\title{
Motivación laboral hacia la investigación científica del profesional de enfermería asistencial en un hospital nacional
}

\author{
Vanessa Camacho-Avalosa, Veronica Escalante-Osorio 1,a, Lidia Quispe Pardo 2,a, \\ Maria del Carmen Salazar Garcia 3,a
}

\section{RESUMEN}

Objetivo: determinar la motivación laboral hacia la investigación científica que presenta el profesional de enfermería asistencial en el Hospital Nacional Arzobispo Loayza. Material y método: estudio descriptivo de corte transversal, constituido por una muestra de 211 profesionales enfermeros que cumplieron con los criterios de selección. Se aplicó un cuestionario tipo Likert que comprende 24 ítems, el cual fue validado mediante juicio de expertos y prueba piloto. La información se procesó y analizó en el software estadístico SPSS versión 21 para obtener tablas de frecuencias absolutas según la variable requerida. Resultados: un $66.3 \%$ indica sentirse motivado a realizar investigación científica como una forma de alcanzar el crecimiento profesional, así como un $43.6 \%$ no realiza investigación porque afirma que implica mayor responsabilidad en su actividad profesional. Además, un $46.9 \%$ del profesional de enfermería afirma que el ambiente físico donde labora no es el adecuado para realizar investigación científica. Conclusiones: la motivación laboral intrínseca está relacionada principalmente con el desarrollo personal, el logro y el crecimiento profesional; y los indicadores con mayor porcentaje obtenido de la motivación laboral extrínseca son condiciones del ambiente físico, el bajo salario, la falta de recursos materiales, y la sobrecarga laboral dentro del horario de trabajo quienes dificultan realizar investigación.

Palabras clave: motivación, investigación en enfermería, Enfermería práctica.

\section{Work motivation towards scientific research professional nursing care in a national hospital}

\begin{abstract}
Objective: To determine the work motivation towards the scientific research presented by the professional nursing care center at National Hospital. Material and Method: A descriptive cross-sectional study, a sample consisting of 203 professional nurses who met the selection criteria. Likert questionnaire comprising of 24 items, which was validated by an expert opinion and the pilot test was applied. The information is processed and analyzed in the statistical software SPSS version 21 for tables of absolute frequencies to the required variables. Results: $66.3 \%$ of the report felt motivated to conduct scientific research as a way to achieve professional growth and $43.6 \%$ was no conducted because it said that it implies greater responsibility for their work. In addition, $46.9 \%$ of the nurses say that the physical environment where they work is not adequate for scientific research. Conclusions: The intrinsic work motivation is primarily related to personal development, achievement and professional growth; indicators obtained with the highest percentage of extrinsic work motivation are conditions of the physical environment, low wages, lack of material resources, and the work overload of the difficult work schedule of than who conducted the research.
\end{abstract}

Keywords: Motivation, Nursing Research, Nursing Practice.

Clínica San Juan de Dios. Lima, Perú.

Clínica Javier Prado. Lima, Perú.

Facultad de Enfermería, Universidad Peruana Cayetano Heredia. Lima - Perú.

Licenciada en Enfermería. 


\section{INTRODUCCIÓN}

La investigación científica en las decisiones de salud constituyen hoy en día un reto no sólo para organizaciones de nivel internacional como la Organización Mundial de Salud (1) sino también para nuestro país, como lo refiere el presidente del Colegio de Enfermeras (os) del Perú, quien indica que la investigación en enfermería es baja, sin la inversión suficiente y con el agravante, que la escasa producción científica es poco conocida por la comunidad científica, la ciudadanía y la misma profesión, debido a que la sistematización y difusión es precaria, pobremente utilizada y validada (2).

Enfermería definida como una profesión científica y humanista, realiza una práctica basada en evidencia científica que fomenta e incrementa el conocimiento para fortalecer las actitudes del profesional y lograr con mayor efectividad los cuidados brindados en beneficio de los usuarios (3). Asimismo, por las características de sus actividades identifican diversos problemas de toda índole, los mismos que serán resueltos de acuerdo a la disponibilidad de recursos. Para garantizar y asegurar la continuidad de los cuidados, la enfermera(o) debe estar capacitado y actualizado permanentemente teniendo como referencia los resultados de las investigaciones más recientes en su área de competencia (4).

Es necesario hacer uso de los recursos en forma eficiente sin afectar la calidad y teniendo una definición clara de las prioridades en la producción de conocimiento de enfermería, de tal forma que respondan tanto a la necesidad de fundamentar la ciencia de enfermería, como a las necesidades en salud (5).

La práctica de la atención de salud basada en evidencia es un imperativo para asegurar una atención de calidad costo efectivo y es a la vez un distintivo de la enfermería profesional, y en especial en aquellas regiones en que los recursos son escasos. Las enfermeras deben ser capaces de identificar y utilizar la mejor evidencia en su ejercicio profesional. Cuando falte evidencia o ésta sea débil, las enfermeras deben ser capaces de generar el conocimiento necesario para suplir esta falencia (6).

Morán y Egúsquiza mencionan que existe falta de motivación del personal para dedicarse a la investigación, y además señalaron que los mismos profesionales no consideran investigar como parte de su actividad laboral, prevaleciendo como principal obstáculo la falta de estímulo de los jefes de servicios para poder investigar (7).

Según Triviño y col. en una publicación de análisis que se realizó sobre paradigmas de la investigación de enfer- mería, plantean que la desmotivación por el desarrollo de la investigación dificulta procesos de estudio y comprensión de los paradigmas en investigación (8).

La motivación laboral según Frederick Herzberg es un proceso que activa e indica el nivel de compromiso laboral, orienta, dinamiza y mantiene el comportamiento de los individuos hacia la realización de objetivos esperados, y al no presentarse afecta directamente el desempeño del trabajador (9). Frederick afirma que la motivación se genera por la búsqueda de la satisfacción óptima de ciertas necesidades, y define dos tipos de necesidades que afectan de manera diversa el comportamiento humano: factor higiénico o extrínseco y factor motivacional o intrínseco (10).

Si se conoce que enfermería al investigar genera sus propios conocimientos, base esencial para desarrollar su objeto disciplinario, además de constituir una parte vital de la práctica clínica y por ende beneficio directo al desarrollo de la profesión, entonces no se explica la razón por la cual la enfermera(o) asistencial no realiza investigaciones dentro de su ámbito laboral (11).

Actualmente, ha aumentado la presión para que enfermería realice investigaciones y publicaciones; sin embargo, la evidencia señala que son escasas y dicha problemática se identifica en diversas investigaciones internacionales y nacionales (12). Considerando lo mencionado, las investigaciones realizadas dan a conocer que la razón principal de la escasa investigación científica en el profesional de enfermería, se debe a la deficiente motivación laboral (13).

Es por ello, que el estudio de investigación surge con el objetivo de determinar la motivación laboral hacia la investigación científica que tiene el profesional de Enfermería asistencial en un Hospital Nacional.

\section{MATERIAL Y MÉTODOS}

El estudio fue descriptivo de corte transversal, se realizó en un Hospital Nacional, institución de categoría de salud III -1, situada en el distrito del Lima, Perú. Se desarrolló en los servicios de hospitalización, consultorios externos, sala de operaciones y estrategias sanitarias, con excepción de los servicios de Emergencia, y Unidad de Cuidados Intensivos (UCI).

La población de estudio estuvo conformada por 421 enfermeros asistenciales que laboran en el hospital, entre contratados y nombrados, que pertenecen a los diferentes servicios del nosocomio. No fue considerado el personal de enfermería administrativo (65), enfermeras de UCI 
(48) y Emergencia (53), según registro proporcionado por la propia institución. La muestra conformada por 203 enfermeros asistenciales (según la fórmula estadística), pero se aplicó el instrumento a 211 enfermeros asistenciales para evitar el factor pérdida al momento de contestar la encuesta. Luego, para conocer quienes participarían del estudio se aplicó el muestreo aleatorio simple, utilizando la tabla de números aleatorios, donde se obtuvo un grupo de 211 enfermeros asistenciales que cumplieron con los criterios de inclusión enfermeros que realizaban labor asistencial en el Hospital Nacional y el criterio de exclusión enfermeros que ocupaban cargos administrativos o de jefatura.

Para la recolección de datos se utilizó la técnica de la encuesta. El instrumento aplicado fue un cuestionario, teniendo en cuenta la escala ordinal tipo Likert, elaborado por las investigadoras, éste estuvo dividido en dos segmentos: el primero, aborda los datos demográficos de la población de estudio (5 - ítems) como edad, sexo, estado civil, tiempo de servicio que tiene en la institución y nivel académico; y el segundo, está enfocado en aspectos relacionados a la motivación laboral hacia la investigación los cuales se dividieron en: motivación intrínseca (12 ítems) y motivación extrínseca (12 ítems). Para la motivación intrínseca se consideraron preguntas relacionadas a la búsqueda de reconocimiento laboral, desarrollo personal, crecimiento profesional, responsabilidad, autonomía de decisión y logro. Para la motivación extrínseca se elaboraron preguntas relacionadas a las condiciones físicas del ambiente del trabajo, relaciones laborales, salario y beneficios sociales, ascensos, seguridad laboral y políticas de organización.

La validación del instrumento fue a través de juicios de expertos, los cuales fueron profesionales, del área de salud mental; y para la confiabilidad se realizó la prueba piloto en 30 enfermeros asistenciales. El resultado para el coeficiente Alpha de Cronbach tuvo un puntaje de 0.718 , el cual se ubica dentro de una categoría aceptable para el análisis de la consistencia interna del instrumento. Para la recolección de datos se contactó con cada enfermera, se le explico los objetivos del estudio y se les entrego el Consentimiento Informado donde se les informaba todas aquellas consideraciones éticas que involucraba el estudio.

La recolección de datos tuvo una duración de quince días, para lo cual se consideraron los tres horarios de trabajo - mañana, tarde y noche que permitieron completar la muestra total.

La información obtenida, ingreso a una base de datos elaborada por las investigadoras. La información ingresada fue procesada y analizada en distintas fases, para ello se utilizó el software estadístico SPSS versión 21. En un inicio se calcularon los puntajes parciales y totales para luego recodificar algunas de las variables que eran necesarias para calcular así las frecuencias y porcentajes respectivos y ser presentados en gráficos de barras simples.

\section{RESULTADOS}

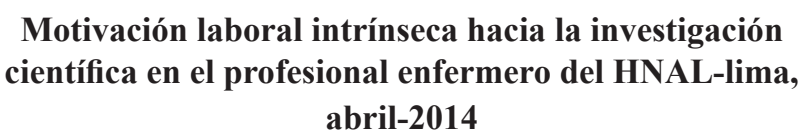

\section{GRÁFICO 1}

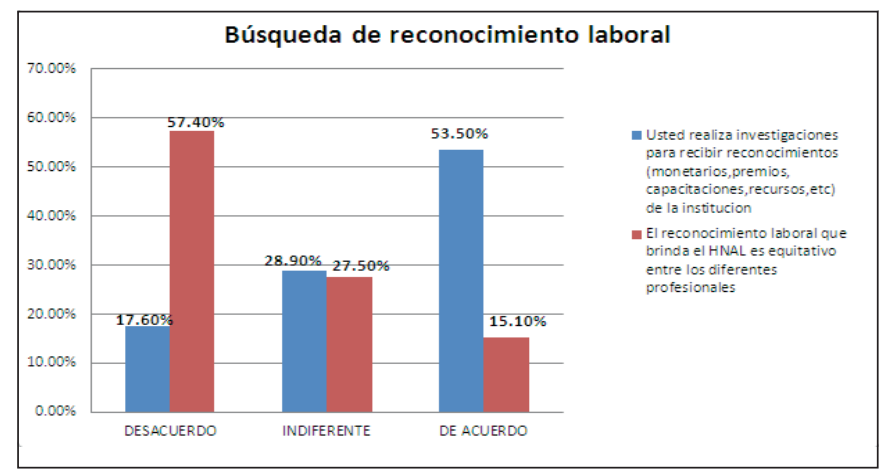

Se observa que el $53.5 \%$ está de acuerdo con realizar investigaciones para recibir reconocimientos por su institución hospitalaria. Pero el $57.4 \%$ está en desacuerdo con el reconocimiento laboral que brinda el Hospital Nacional Arzobispo Loayza por considerar que no es equitativo entre los diferentes profesionales.

\section{GRÁFICO 2}

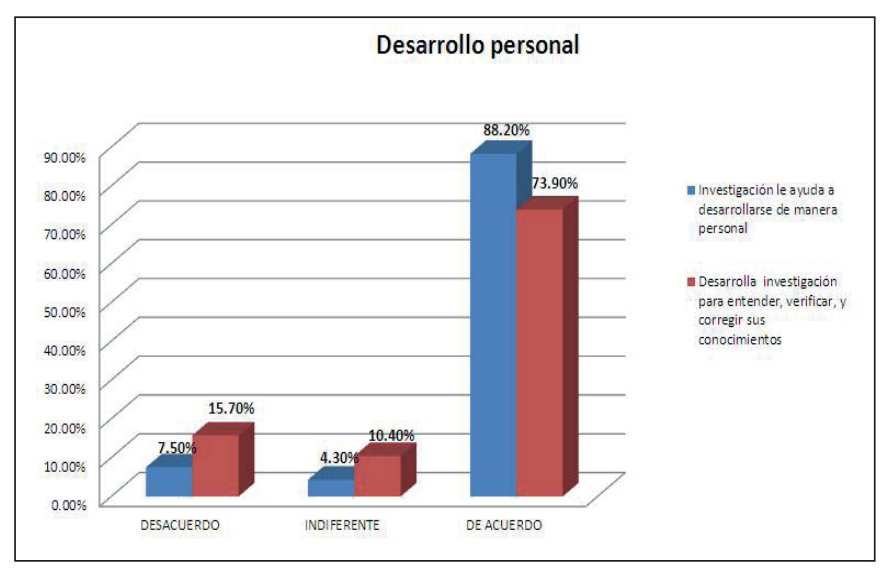

Se observa que el $88.2 \%$ considera que la investigación le ayuda a desarrollarse de manera personal; así como, el $73.9 \%$ está de acuerdo con desarrollar investigación para entender, verificar y corregir sus conocimientos. 


\section{GRÁFICO 3}

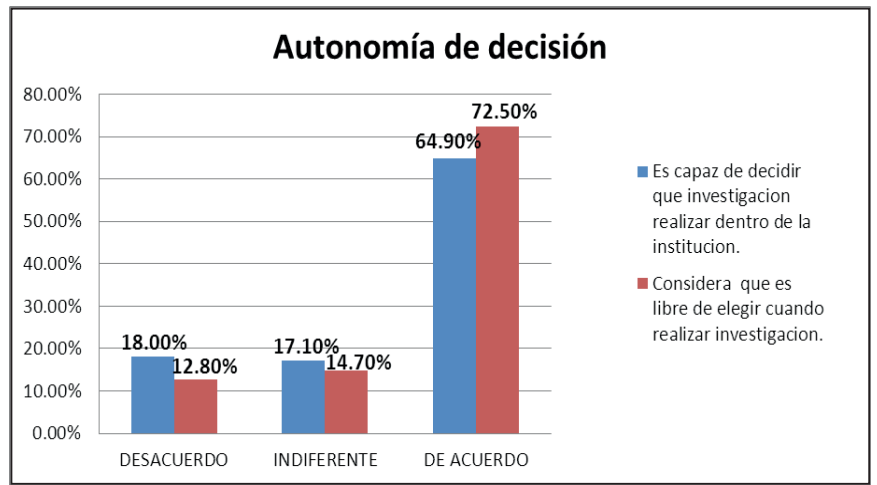

Se observa que el 64.9\% indica que es capaz de decidir qué investigación realizar dentro de la institución hospitalaria, y el $72.5 \%$ es libre de elegir cuando realizar investigación.

\section{GRÁFICO 4}

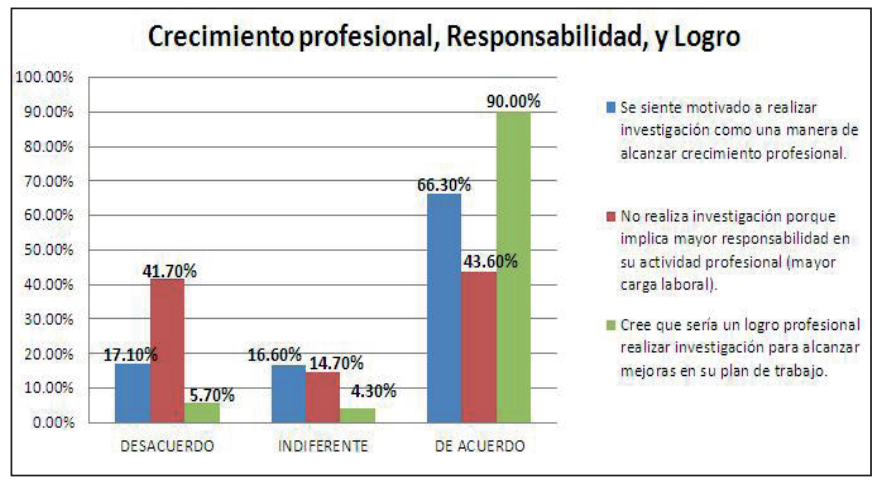

El 66.3\% se siente motivado a investigar como una forma de alcanzar el crecimiento profesional, y además el $90 \%$ cree que sería un logro profesional realizar investigación para alcanzar mejoras en su plan de trabajo. Pero el $43.6 \%$ no realiza investigación porque implica mayor responsabilidad en su actividad profesional (mayor carga laboral).

\section{GRÁFICO 5}

Motivación laboral extrínseca hacia la investigación científica en el profesional enfermero del HNAL-Lima, abril-2014

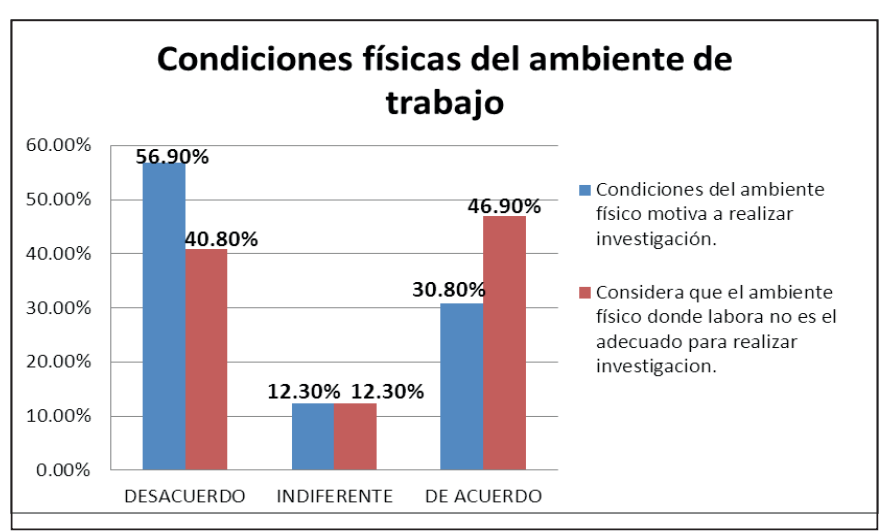

E1 56.9\% señala que el ambiente físico no motiva a realizar investigación, y además el 46.9\% considera que el ambiente físico donde labora no ser el adecuado para realizar investigaciones científicas

\section{GRÁFICO 6}

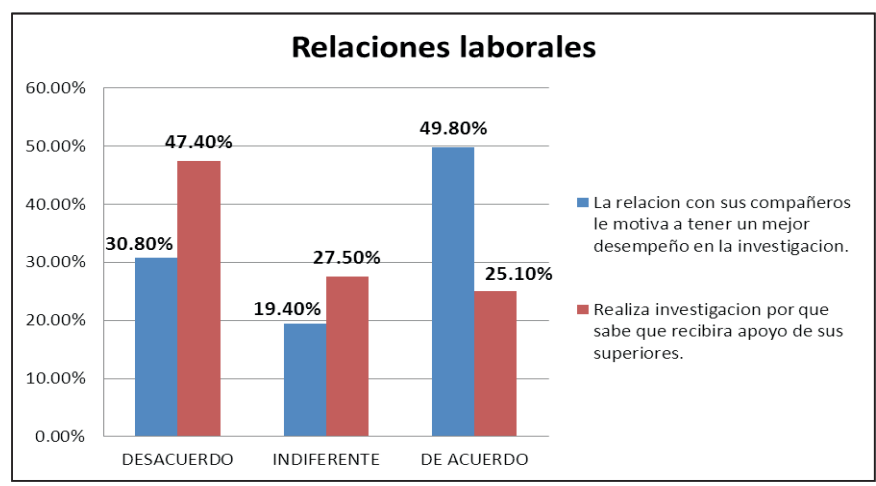

El 49.8\% señala que sus compañeros de trabajo les motivan a tener un mejor desempeño en la investigación, pero también se observa que el $47.4 \%$ está en desacuerdo con realizar investigación por considerar que no recibirá apoyo por parte de sus superiores.

\section{GRÁFICO 7}

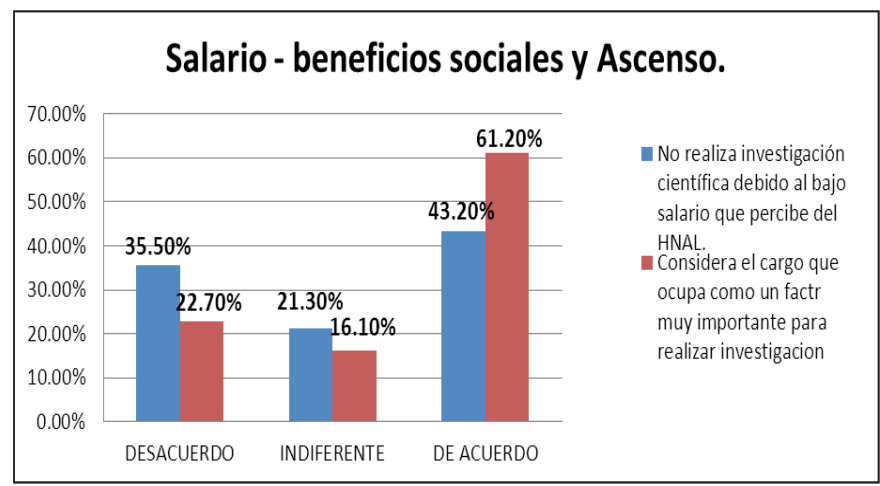

Se observa que el $61.2 \%$ considera el cargo que ocupa como un factor muy importante para realizar investigación, pero respecto al indicador de salario el $43.2 \%$ no realiza investigación debido al bajo salario que percibe del HNAL.

\section{GRÁFICO 8}

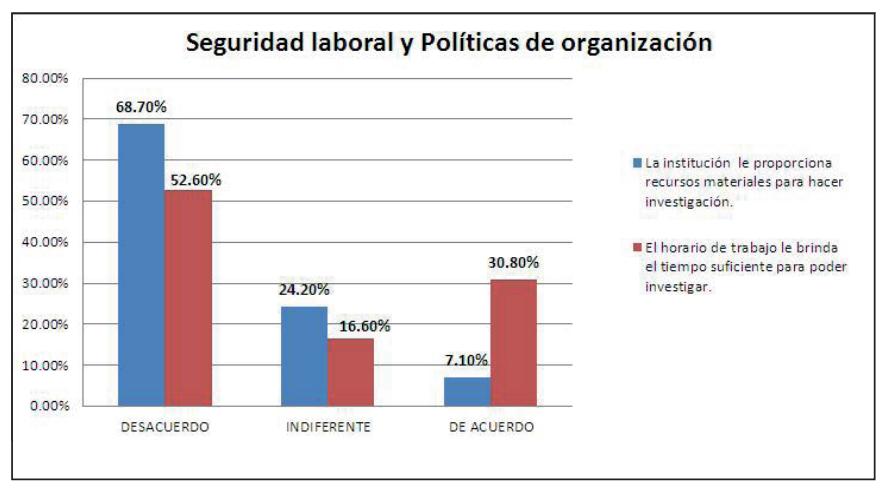


Se observa que el $68.7 \%$ señala que el HNAL no le brinda recursos materiales (material de escritorio básico) para hacer investigación, y además el $52.6 \%$ afirma que el horario de trabajo no le brinda tiempo suficiente para poder investigar.

\section{DISCUSIÓN}

Respecto a la motivación laboral intrínseca hacia la investigación científica un 53.5\% (gráfico 1) está de acuerdo con realizar investigación para recibir reconocimiento por parte de la institución; mientras que un $57.4 \%$ señala estar en desacuerdo con el reconocimiento laboral que brinda la institución por no considerarlo equitativo entre los diferentes profesionales de la salud que realizan investigación. Respecto a lo encontrado, la investigación realizada por Cepeda y col. señalan que el $68.5 \%$ piensa que enfermería no recibe los mismos apoyos externos (becas, premios, subvenciones, publicaciones, ni reconocimientos) que otros profesionales para investigar; a pesar de que el $89.7 \%$ de los profesionales de enfermería consideran que están igual de capacitados que otros profesionales de salud para realizar un estudio de investigación (15).

Esto señalaría que la enfermera asistencial tiene entre sus principales objetivos alcanzar algún tipo de reconocimiento y/o distinción que gratifique el aporte investigativo que podría brindar como enfermera asistencial; sin embargo, percibe que el reconocimiento de su institución no cumple sus expectativas y esto influiría negativamente en su desempeño investigador como enfermera.

En el segundo indicador, si la investigación le ayuda a desarrollarse de manera personal el $88.2 \%$ considera que si y el $73.9 \%$ investiga para entender, verificar y corregir sus conocimientos. Esto demuestra que la enfermera asistencial reconoce la importancia que tiene investigar para el incremento de sus habilidades, del pensamiento crítico y la capacidad de alcanzar el perfeccionamiento de sus conocimientos a través del quehacer investigativo.

Así mismo se observa el $66.3 \%$ se siente motivado a realizar investigación como forma de alcanzar un crecimiento profesional y un $90 \%$ cree que sería un logro profesional realizar investigación científica para alcanzar mejoras en su plan de trabajo. Resultados semejantes son los hallados por Ortuño y col. quienes indican que un $100 \%$ de encuestados manifiesta la importancia y el beneficio que la investigación tiene para la práctica profesional, y ello se sustenta en un mayor conocimiento, debido a que consideran la investigación como una función inherente de la profesión y desarrollo personal (14).
De igual modo, Cepeda y col. señalan que los enfermeros se sienten capacitados para decidir los cuidados del paciente y estarían dispuestos a colaborar con otros profesionales a investigar, además de considerarlo como necesario para mejorar la práctica y alcanzar el logro profesional (15). Así también Espejo y col. muestran similitud entre los resultados al identificar que los factores laboral y académico, influyen a nivel de producción científica (16).

Pero el $43.6 \%$ no realiza investigación porque implica mayor responsabilidad en su actividad profesional lo que se traduce como una mayor carga laboral. Cepeda y col. mencionan resultados similares, donde se señala que un problema importante es la elevada carga asistencial que tiene la enfermera, donde un $65 \%$ refiere que esto hace difícil la investigación durante la jornada laboral (15).

Dentro de la motivación laboral extrínseca se muestra el indicador de condiciones físicas del ambiente de trabajo, donde se puede observar que el $56.9 \%$ refiere que el ambiente físico no les motiva a realizar investigación, esto puede deberse a que de las enfermeras señalan que el ambiente donde desempeñan su labor no es el adecuado para realizar investigaciones, resultado similar al encontrado en el estudio de Cogollo y Gómez, donde respecto a las condiciones físicas del puesto de trabajo, evidencia que las enfermeras asistenciales laboran en condiciones físicas inadecuadas como: el 31\% labora en un espacio reducido y un $15 \%$ refiere estar sometido bajo niveles de alto ruido en su área de trabajo (17). Frederick Herzberg menciona en su Teoría Bifactorial, que las inadecuadas condiciones físicas del ambiente de trabajo influyen de forma negativa para el desarrollo de un óptimo trabajo y esto dificulta al profesional a realizar investigación por considerar su entorno como inadecuado. Sin embargo, esta situación no impide investigar, puesto que como profesión se debe poner al servicio de la sociedad la producción y el uso responsable del conocimiento y esto se efectúa al investigar (20).

Con respecto al indicador de salario un considerable $43.2 \%$ afirma no realizar investigaciones por el bajo salario que percibe de su institución y si tenemos en cuenta que el salario es uno de los motivadores más eficaces; como plantea Locke; y es valorado como un símbolo de realización, de reconocimiento de status, además de ser punto de referencia por medio del cual las enfermeras comparan su valor con otros profesionales. Estaríamos viendo que estos resultados forman parte de una problemática nacional en el cual observamos de manera continua que el profesional de salud siente que su salario está por debajo de las labores que realiza. 
A esto sumariamos que un $68.7 \%$ de enfermeras indican que la institución hospitalaria no le proporciona recursos materiales (elementos de escritorio básicos) para realizar investigación, resultado similar se encontró en el estudio de Cepeda y col quienes muestran en su estudio que un $47.1 \%$ considera que para investigar se necesita recursos económicos difíciles de conseguir (15). Cogollo y Gómez en su estudio señalan que un $34 \%$ de las enfermeras asistenciales consideraron que los equipos y materiales de trabajo eran incompletos e inseguros para realizar investigación (17). Asimismo, se observa que un $61.2 \%$ de enfermeras consideran el cargo que ocupa como un factor muy importante para realizar investigación.

Estos indicadores mencionados como salario, materiales de escritorio que no proporciona el hospital, así como el ambiente inadecuado; conducen a que las enfermeras tengan que proveerse de recursos propios para investigar lo cual estaría sumándose a un obstáculo más que tendría que afrontar el profesional de enfermería. Ya que ellas consideran el aspecto económico, evidenciado por este estudio como un ente muy limitante para investigar, debido a los escasos recursos que poseen.

Por otro lado observamos que el 52.6\% indica que el horario de trabajo no le brinda tiempo suficiente para poder investigar; puesto que, no se dispone de un tiempo específico dedicado a la investigación, debido a una elevada presión asistencial dentro de la jornada laboral y al concluir con esas actividades, las responsabilidades personales y familiares suponen otra disminución de tiempo que dificultan el desarrollo de la investigación científica.

Resultado similar a lo descrito por Díaz y col. en su estudio de investigación, en el que muestra que un $76 \%$ de enfermeras asistenciales no participó, ni realizó investigación científica porque no disponían de tiempo y que no contaban con los estímulos y facilidades por parte de la institución y del departamento de enfermería (18). Otro resultado similar es el de Ortuño y col. quienes encuentran que un $60.6 \%$ del personal de enfermería asistencial reconocen que existe falta de tiempo para desarrollar la investigación (14).

Es que el indicador tiempo estaría ligado a distintos elementos desencadenantes que conllevan a su escases; como el escaso suministro de servicios, reducido recurso humano, infraestructura inadecuada e incluso que enfermería no dispone de un tiempo específico dedicado a la investigación; lo cual conducen a una elevada presión asistencial dentro de sus horas de trabajo (17), sería por ello que el $43.6 \%$ del personal indica que no investiga por la elevada carga asistencial que esto le originaria, convirtiéndose la suma de todos ellos en un gran elemento obstaculizante para realizar investigación de la enfermería asistencial.

No se evidenciaron estudios referentes a la motivación laboral medida a través de intrínseco y extrínseco que permitan contrastar con los resultados obtenidos. Sin embargo, se puede mencionar como referencia a Valverde, que en su estudio menciona que un $70.8 \%$ de enfermeras muestran una actitud favorable hacia la investigación (21).

Al conocer los resultados de la presente investigación, es necesario señalar que existe la motivación implícita de los profesionales enfermeros por realizar investigación científica, y esto de alguna manera debe seguir fortaleciéndose para tener resultados concretos.

Por ello, en el ámbito laboral es importante conocer las causas que estimulan la acción humana, ya que mediante el manejo de la motivación, entre otros aspectos, los jefes de servicios pueden operar estos elementos a fin de que su organización funcione adecuadamente y los miembros se sientan más satisfechos (19).

\section{REFERENCIAS BIBLIOGRÁFICAS}

1. Organización Panamericana de la salud (OMS). La investigación en salud como instrumento para mejorar la salud pública. Pan American Health Organization; 2004. Disponible en: http://www.eventos. bvsalud.org/agendas/BVS-COR/public/documents/ analia_porras-154419.pdf.

2. Mendigure Fernández J. Colegio de Enfermeras (os) del Perú - CICEP. [Internet]. [citado 2014 Enero 14] Disponible en: http://www.cep.org.pe/cicep/revista/ volumen1/editorial.pdf.

3. Orellana A, Sanhueza O. Competencia en investigación en enfermería. [Internet] 2011. [citado 2014 Mayo 20] Disponible en: http://www. scielo.cl/scielo.php?script=sci_arttext\&pid $=$ S0717-95532011000200002.

4. Estefo S, Paravic T. Enfermería en el rol de gestora de los cuidados. [Internet] 2010. [citado 2014 Mayo 10] Disponible en: http://www. scielo.cl/scielo.php?script=sci_arttext\&pid $=\mathrm{S} 0717-95532010000300005$.

5. Castrillón M. Tendencias y prioridades de Investigación en Enfermería. [Internet] 2004. [citado 2014 Enero 10] Disponible en: http://www.scielo.br/scielo. 
php?script $=$ sci_arttext\&pid $=$ S010411692004000400 002\&lng $=$ es\&nrm $=$ iso\&tlng $=$ es.

6. Harrison L, Hernández A, Cianelli R. Competencias en investigación para diferentes niveles de formación de enfermeras: una perspectiva latinoamericana. [Internet] 2005. [citado 2014 Febrero 20] Disponible en: http://www.scielo.cl/scielo.php?script=sci_arttext\&p $\mathrm{id}=\mathrm{S} 0717-95532005000100007$.

7. Moran F, Egusquiza L. Motivación y nivel de satisfacción laboral de la enfermera en el servicio de infectología y neumología del Instituto Nacional de Salud del Niño. [Tesis]. Lima - Perú. Universidad Peruana Cayetano Heredia. 2003. [Citado 2014 Enero 20].

8. Triviño Z, Sanhueza O. Paradigmas de investigación en enfermería. [Internet] 2005. [citado 2014 Febrero 22] Disponible en: http://www. scielo.cl/scielo.php?script=sci_arttext\&pid $=$ S0717-95532005000100003.

9. Medina A, Gallegos C, Lara P. Motivación y satisfacción de los trabajadores y su influencia en la creación de valor económico en la empresa. [Internet] 2008. [citado 2014 Junio 4] Disponible en:

http://www.scielo.br/pdf/rap/v42n6/09.pdf.

10. López J. Motivación laboral y gestión de recursos humanos en la teoría de Frederick Herzberg. [Internet] 2005. [citado 2014 Junio 4] Disponible en: http://sisbib.unmsm.edu.pe/bibvirtualdata/publicaciones/administracion/n15_2005/a04.pdf.

11. Urra E. Avances de la ciencia de enfermería y su relación con la disciplina. [Internet] 2009. [citado 2014 Marzo 15]Disponibleen:http://www.scielo.cl/scielo. php?pid=S0717-95532009000200002\& script $=$ sci arttext.

12. Ibarra T, Noreña A. Visibilidad de la disciplina enfermera: el factor mediático de la investigación. [Internet] 2011. [citado 2014 Mayo14] Disponible en: http://scielo.isciii.es/scielo.php?script=sci_arttext\& pid $=$ S1132-12962011000300009.

13. Yagüe J. Enfermería basada en la evidencia: una visión desde la práctica clínica. [Internet] 2008. [citado 2014 Febrero 14]. Disponible en: http://www. index-f.com/evidentia/n22/e6802.php

Fecha de recibido: 03 de Noviembre de2014

Fecha de aceptación: 15 de Diciembre de 2014.
14. Ortuño I, Posada P, Fernández E. Actitud y motivación frente a la investigación en un nuevo marco de oportunidad para los profesionales de enfermería. [Internet] 2013. [citado 2014 Marzo 28] Disponible en: http://scielo.isciii.es/scielo.php?pid=S1132$12962013000200004 \&$ script=sci_arttext.

15. Cepeda J, San Román J, Álvarez C. Actitud y motivación de la enfermería de Castilla y León hacia la investigación. [Internet] 2010. [citado en 2014 Marzo 14] Disponible en: www.revistaenfermeriacyl. com/index.php/revistaenfermeriacyl/.../48.

16. Espejo S, Hijar G, Sandoval D. Factores que influyen en la participación de la producción científica del personal de enfermería que elabora en el centro médico naval. (Tesis). Lima - Perú. Universidad Peruana Cayetano Heredia. 2001 [Citado 2014 Enero 20].

17. Cogollo Z, y Gomez E. Condiciones laborales en enfermeras de Cartagena, Colombia. 2010 Enero - Junio. [citado en 2014 Marzo 14]. Disponible en: http://www.scielo.org.co/pdf/aven/v28n1/ v28n1a04.pdf.

18. Díaz M, Quispe C, Santibáñez Y. Actitudes y algunos factores que influyen en la realización de investigación científica en enfermeras asistenciales del hospital general nacional Arzobispo Loayza. [Tesis]. Lima - Perú. Universidad Peruana Cayetano Heredia. 1994. [citado 2014 Febrero15].

19. Guidarelli R. Producción científica: características motivos y factores expresados. [Internet] 2007. [citado 2014 Abril 14]. Disponible en: http://www.intramed.net/contenidover.asp?contenidoID $=34728$.

20. Manso J. El legado de Frederick Irving Herzberg. [Internet] 2002. [citado 2014 Enero 30]. Disponible en: http://www.redalyc.org/pdf/215/21512808.pdf.

21. Valverde N. Actitud de las enfermeras hacia la investigación y factores que intervienen en su realización en el Hospital Nacional Daniel Alcides Carrión. [Tesis]. Lima - Perú. Universidad Nacional Mayor de San Marcos. 2005. [citado 2014 Febrero15] Disponible en: http://cybertesis.unmsm.edu.pe/bitstream/ cybertesis/1052/1/valverde_cm.pdf. 